\title{
Antimicrobial Susceptibility Patterns of Enterobacteriaceae Isolated from Stool Samples at a Semi-urban Teaching Hospital
}

\author{
Charles John Elikwu" ${ }^{1,2, *}$ Emmanuel Olushola Shobowale ${ }^{1,2}$, Victor Ugochukwu Nwadike ${ }^{1}$, \\ Babatunde Tayo ${ }^{1}$, Chika Celen Okangba ${ }^{1}$, Opeoluwa Akinyele Shonekan ${ }^{1}$, \\ Azubuike Chidiebere Omeonu ${ }^{1}$, Bibitayo Faluyi ${ }^{1}$, Pearl Ile ${ }^{1}$, Adebola Adelodun ${ }^{1}$, \\ Adebusola Popoola ${ }^{1}$, Maxwell Mubele ${ }^{1}$ \\ ${ }^{1}$ Department of Medical Microbiology \& Parasitology, Ben Carson School of Medicine, Babcock University, Ilisan-Remo, Nigeria \\ ${ }^{2}$ Department of Medical Microbiology \& Parasitology, Babcock University Teaching Hospital, Ilisan-Remo, Nigeria
}

Email address:

elikwuc@babcock.edu.ng (C. J. Elikwu)

\section{To cite this article:}

Charles John Elikwu, Emmanuel Olushola Shobowale, Victor Ugochukwu Nwadike, Babatunde Tayo, Chika Celen Okangba, Opeoluwa Akinyele Shonekan, Azubuike Chidiebere Omeonu, Bibitayo Faluyi, Pearl Ile, Adebola Adelodun, Adebusola Popoola, Maxwell Mubele. Antimicrobial Susceptibility Patterns of Enterobacteriaceae Isolated from Stool Samples at a Semi-Urban Teaching Hospital. American Journal of Biomedical and Life Sciences. Vol. 3, No. 6, 2015, pp. 127-130. doi: 10.11648/j.ajbls.20150306.15

\begin{abstract}
Enterobacteriaceae cause a wide range of diseases including urinary tract infections, respiratory tract infections, sepsis, and gastroenteritis. They are the most frequently recovered pathogens from clinical samples and have varying susceptibility patterns. The study set out to determine the susceptibility profile of enterobacteriaceae species at the Babcock University Teaching Hospital. Enterobacteriaceae were identified using the Microbact 12A kit (Oxoid UK) and susceptibility was determined with the modified Kirby-Bauer Method in line with CLSI 2014 guidelines. Escherichia coli the main isolate was $100 \%$ susceptible to Piperacillin/Tazobactam, $94 \%$ susceptible to Amikacin, $76.5 \%$ susceptible to both Ampicillin/Sulbactam and Ceftazidime, 70.6\% susceptible to Ceftriaxone and Meropenem, 67\% susceptible to Ciprofloxacin, $58 \%$ Susceptible to Gentamicin and $23.5 \%$ susceptible to Amoxicillin/Clavulanic acid. Antibiotic resistance among Enterobacteriaceae is on the rise in Babcock University Teaching Hospital. Measures should be put in place to prevent more resistance and to prevent spread of resistant strains.
\end{abstract}

Keywords: Antimicrobial Resistance, Escherichia Coli, Enterobacteriaceae, Babcock University

\section{Introduction}

The Enterobacteriaceae are a large heterogeneous family of Gram-negative rods whose natural habitat is the intestinal tract of humans and animals. They are facultative anaerobes or aerobes, ferment a wide range of carbohydrates, possess a complex antigenic structure, and produce a variety of toxins and other virulence factors. Members of the Enterobacteriaceae are rod-shaped, and are typically 1-5 $\mu \mathrm{m}$ in length [1-4].

They appear as small grey colonies on blood agar. Like other proteobacteria, enterobacteria have Gram-negative stains and they are facultative anaerobes, fermenting sugars to produce lactic acid and various other end products. Most also reduce nitrate to nitrite, although exceptions exist (e. g.
Photorhabdus). Unlike most similar bacteria, enterobacteriaceae generally lack cytochrome $\mathrm{C}$ oxidase, although there are exceptions (e. g. Plesiomonas shigelloides). Most have many flagella used to move about, but a few genera are non-motile. They are also non sporeforming while Catalase reactions are positive [5-7].

They are also called enteric bacteria or coliforms. Some are harmless symbionts like Escherichia coli while many are familiar pathogens, such as Salmonella spp, Yersinia pestis, Klebsiella pneumoniae and Shigella spp. Other diseasecausing bacteria in this family include Proteus spp, Enterobacter spp, Serratia spp, and Citrobacter spp [8-10].

Enterobacteriaceae cause a wide range of diseases including urinary tract infections, respiratory tract infections, sepsis and gastroenteritis. Several enterobacteriaceae strains 
have been isolated which are resistant to antibiotics including the carbapenems, which are often claimed to be "the last line of antibiotic defense" against resistant organisms. For instance, some Klebsiella pneumoniae strains are carbapenem resistant [11-15].

Antibiotic misuse in humans and animals, hospital crossinfection, the food chain, trade and human migration seem to have contributed to the recent dissemination of resistant enterobacteriaceae outside hospitals, although the role of these factors is variable and linked to particular epidemiological situations. Within hospitals or other institutions, these bacteria commonly are transmitted by personnel, instruments, or parenteral medications. Their control depends on hand washing, rigorous asepsis, sterilization of equipment, disinfection, restraint in intravenous therapy, and strict precautions in keeping the urinary tract sterile i. e closed drainage [16-21].

Several risk-factors for enterobacteriaceae infections have been identified especially among patients on admission. These include; critically ill patients or severely debilitated residents especially those having prolonged hospital or ICU unit stay, invasive procedures like indwelling catheter, central venous catheter, gastrostomy, tracheostomy, endotracheal or nasogastric tube. Patients that have had prolonged stay in health care facility are totally dependent on healthcare workers and those that develop decubitus ulcers are also predisposed to acquiring these infections [22-26]. Treatment with other antibiotics especially third generation Cephalosporins, Quinolones, TrimethoprimSulphamethaxazole, Aminoglycosides appear to be a significant risk-factor for the selection of infections [27, 28].

Resistant enterobacteriaceae have huge impact on patient care as infections from these organisms lead to longer hospital stay, increased healthcare costs, and higher mortality rates and emergence and spread in the community. This study had generated a baseline data on the antibiotic susceptibility profile of enterobacteriaceae isolated from stool samples in our institution.

This study was aimed to determine the susceptibility profile of enterobacteriaceae species at the Babcock University Teaching Hospital. These are to acquire data on the antimicrobial susceptibility profile of enterobacteriaceae isolates under standardized conditions also to know the risk factors for acquisition of such pathogens.

\section{Methods}

This was a prospective descriptive cross sectional study in Babcock University Teaching Hospital Nigeria carried out between February and June of 2015. The Hospital is a 140 bed tertiary health institution which provides healthcare services to the people of Ogun state and neighbouring Lagos and Ondo states in South West Nigeria.

The study population consisted of patients from Accident and Emergency, Paediatrics, Surgery, Medicine, Obstetrics and Gynaecology wards as well community medicine clinic patients. The study was conducted at the Research laboratory of the department of Medical Microbiology using a sample size of 50 participants.

Rectal swabs and frank stool samples were collected from participants by resident doctors and medical students in the concerned departments for culture and sensitivity. Specimens were collected using swab sticks and universal bottles.

The specimens were inoculated on selective and nonselective media. The specimens were inoculated on MacConkey agar without salt and incubated at $35-37^{\circ} \mathrm{C}$ for 18-24hrs. A discrete colony was picked and emulsified on normal saline on a clean microscope slide. Gram negative, oxidase negative organisms were emulsified in normal saline in a bijou bottle. The turbidity was matched with a suspension of 0.5 McFarland's standard and corrected to standard. Thereafter four drops of the suspension were dispensed into each Microbact 12A strip using a $2 \mathrm{ml}$ syringe, mineral oil was added to it and it was read within the first 3 minutes. The strip was then incubated. The rest of the suspension was cultured on Mueller-Hinton agar and used to antimicrobial susceptibility.

Antibiotic susceptibility by the Modified Kirby Bauer disk diffusion method was done on the Mueller-Hinton agar following the Clinical and Laboratory Standards Institute (CLSI).

The following antibiotics were tested: ceftriaxone (CRO), ampicillin sulbactam (SAM 30), ciprofloxacin (CIP), amikacin (AK30), augmentin (AMC), gentamycin (GN), piperacillin/tazobactam (TZP 10), ceftazidime (CAZ), meropenem (mem), imipenem (IMI 10) and ampicillin (Amp10). The diameter of the zone of inhibition was measured with a calibrated meter rule and interpreted with reference to standard interpretative CLSI charts.

Ethical approval was obtained from Babcock University Health Research Ethics Committee. The participants were given an informed consent form attached to the questionnaire clearly stating the benefits of the research and the absence of risks and signed the consent form as a proof of consent. The research was only carried out if the participants gave their consent. The participants were allowed to withdraw from the research at any point in time.

\section{Results}

The results of the in vitro activity of the antibiotics against the tested enterobacterieceae isolates are as presented in table 1. These results show that Escherichia coli isolates were $100 \%$ susceptible to piperacillin/tazobactam, $94 \%$ susceptible to Amikacin, $76.5 \%$ susceptible to ampicillin/sulbactam and ceftazidime, $70.6 \%$ susceptible to ceftriaxone and meropenem, $67 \%$ susceptible to ciprofloxacin, 58\% susceptible to gentamicin and $23.5 \%$ susceptible to amoxicillin/clavulanicacid. The resistance rates among the same isolates are as follows: $100 \%$ to ampicillin, $58.8 \%$ to amoxicillin/clavulanic acid, $17.6 \%$ to ceftazidime and meropenem, $35.3 \%$ to gentamicin, $29.4 \%$ to ciprofloxacin, $11.8 \%$ to ceftriaxone, $5.9 \%$ to ampicillin/sulbactam and amikacin and $0 \%$ to piperacillim/tazobactam. 
Enterobacter aerogenes showed 100\% resistance to ampicillin, amoxicillin/clavulanic, intermediate resistance to piperacillin/tazobactam, gentamicin and ciprofloxacin and was $100 \%$ susceptible to ceftriaxone while Acinectobacter baumanni showed $100 \%$ resistance to ampicillin, amoxicillin/clavulanic acid, intermediate resistance to piperacillin/tazobactam and gentamicin, and was $100 \%$ susceptible to ampicillin/sulbactam, ceftriaxone, ceftazidime, meropenem and Amikacin

Table 1. Antimicrobial susceptibility patterns of isolates from stool samples.

\begin{tabular}{|c|c|c|c|c|c|c|c|c|c|}
\hline Organism & TZP & MEM & CRO & CIP & $\mathbf{C N}$ & SAM & AK & AMC & CAZ \\
\hline E.coli $\mathrm{n}=16$ & 100 & 70.6 & 70.6 & 67 & 58 & - & 94 & 76.5 & 23.5 \\
\hline E.aerogenes $\mathrm{n}=5$ & 60 & 100 & 20 & 40 & 60 & 0 & 40 & 0 & 60 \\
\hline A. baumanii $\mathrm{n}=2$ & 100 & 100 & 50 & 100 & 50 & 0 & 0 & 50 & 50 \\
\hline
\end{tabular}

\section{Discussion}

Antibiotic resistance is a major public health issue and resistance among several members of the family Enterobacteriaceae has been recognized worldwide as a serious threat especially in developing countries. [18] The results presented show the susceptibility profile of various antimicrobials and reveal that resistance in this institution is gradually following the worldwide trend. Of the 50 isolates $36 \%$ were Enterobacteriaceae of which $88.9 \%$ were Escherichia coli, 5.5\% were Enterobacteraurogenes and the remaining 5.5\% were Acinetobacter baumani.

Escherichia coli's role as a known pathogen in multi-drug resistance is well described. In this study, Escherichia coli isolates showed the highest resistance to Ampicillin. The high resistance observed among Escherichia coli isolates could be as a result of the widespread urinary tract infections with various strains which have been exposed to most of the commonly used antibiotics. The common use of this drug for treatment of various infections over time could explain this resistance. This correlates with the previous work carried out by other researchers who reported high incidence among Escherichia coli isolates. [29-32]

Intermediate resistance to amoxicillin/clavulanic acid, ampicillin/sulbactam, ceftriaxone, was recorded, an increase in the MIC - Minimum Inhibitory Concentration could improve susceptibility. Meropenem, Gentamycin, Ciprofloxacin, Ceftazidime also should a level of intermediate resistance. The use of these drugs should be carefully monitored because there is a tendency of these drugs to become very ineffective. Increased doses and use in combinations could also improve the effectiveness and prevent resistance.

Piperacillin/tazobactam showed the highest effectiveness in this study. It is a combination antibiotic containing the extended spectrum penicillin antibiotic, piperacillin and the beta lactamase inhibitor, tazobactam. It is majorly used in intensive care medicine and empirical treatment in febrile neutropenia like after chemotherapy. The indications for the prescription of this drug has excluded largely from being overused and overprescribed thereby protecting its effectiveness. The practice of self-medication which is aided by the availability of antibiotics as over-the-counter medications may contribute to resistance to common drugs as noted in this study.

Unauthorised prescription by unqualified personnel of antibiotics could be a cause of the rising resistance. Some participants in this study were immuno-compromised and on prolonged hospital admission, instrumentation and prolonged antibiotic therapy leading to health-care associated infection with resistant strains. The treatment of livestock, poultry with common antibiotics leads the development of resistance and transfer of these resistant strains to human hosts. [33-38]

\section{Limitations of the Study}

The duration of the study was short giving for a small sample size. A larger sample size collected over a longer period of time could reveal a better picture of the susceptibility profile. Also the study was an in vitro one and is only an estimation of what is obtainable in vivo.

\section{Conclusion}

In conclusion, antibiotic resistance among enterobacteriaceae is on the rise in Babcock University Teaching Hospital. Measures should be put in place to mitigate this scourge and to prevent spread of resistant strains. Future studies are recommended to check trends on susceptibility profiles and strict stewardship measures should be taken.

\section{References}

[1] Williams \& Wilkins (1984). The Gammaproteobacteria. In George M. Garrity, editor. Bergey's Manual of Systematic Bacteriology 2B (2nd ed.). New York: Springer. p. 1108. ISBN 978-0-387-24144-9. British Library no. GBA561951.

[2] Aeschlmann (2003). The role of Multidrug Efflux Pumps in the Antibiotic Resistance of Pseudomonas aeruginosa and other Gram negative bacteria. Pharmacotherapy 22(7): 916924.

[3] Fleming A (1945) Penicillin. Nobel Lecture delivered December 11, 1945.

[4] Fluit ADC, Visser MR, Schmitz FJ (2001) Molecular Detection of Antimicrobial resistance. Clinical Microbiology Reviews 14(4): 836-871.

[5] Forbes BA, Sahm DF, Weissfeld AS (1998) Bailey And Scott's Diagnostic Microbiology, 10th edn. Mosby Inc., St. Louis Missouri, USA. 
[6] Giguère S (2006). Antimicrobial Drug Action and Interaction: An Introduction. Antimicrobial therapy in Veterinary Medicine 4th edn, S Giguère, JF Prescott, JD.

[7] Gillespie SH (2001) Antibotic Resistance in the Absence of selective Pressure. International Journal of Antimicrobial Agents 17: 171-176.

[8] Higgins CF (2007). Multiple Molecular Mechanisms for Multidrug resistance Transporters. Nature 446749-757.

[9] Kolář M, Urbánek K, Látal T (2001) Antibiotic selective pressure and development of bacterial resistance. International Journal of Antimicrobial Agents 17 (5): $357-$ 363.

[10] Kumar A and Schweizer HP (2005) Bacterial Resistance to Antibiotics: Active Efflux and Reduced Uptake. Advanced Drug Delivery Reviews 57: 1486-1513. 17: 357-363.

[11] Lambert PA (2005) Bacterial resistance to Antibiotics: Modified Target Sites. Advanced Drug Delivery Review. 57: 1471-1485.

[12] Levy SB (2002) The Antibiotic Paradox, 2nd edition. Perseus Publishing, USA.

[13] Alekshun MN and Levy S (2007) Molecular Mechanisms of Antibacterial Multidrug Resistance. Cell 128: 1037-1050.

[14] Williams KP, Gillespie JJ, Sobral BWS, Nordberg EK, Snyder EE, Shallom JM, Dickerman AW (2010) "Phylogeny of Gamma-proteobacteria". Journal of Bacteriology 192 (9): 2305-2314.

[15] Martinez JL and Baquero F (2000) Mutation frequencies and antibiotic resistance. Antimicrobial Agents and Chemotherapy 44(7): 1771-1777.

[16] Nordmann P and Poirel L (2005) Emergence of PlasmidMediated Resistance to Quinolones in Enterobacteriaceae. Journal of Antimicrobial Chemotherapy 56: 463-469.

[17] Poole K (2005) Efflux-mediated Antimicrobial Resistance. Journal of Antimicrobial Chemotherapy 56: 20-51.

[18] Roe MT and Pillai SD (2003) Monitoring and Identifying Antibiotic resistance Mechanisms in Bacteria. Poultry Science 82: 622-626.

[19] Rouveix B (2007) Clinical implications of multiple drug resistance efflux pumps of pathogenic bacteria. Journal of Antimicrobial Chemotherapy 59: 1208-1209.

[20] Sköld O (2000) Sulfonamide Resistance: Mechanisms and Trends. Drug Resistance Updates 3: 155-160.

[21] Turnidge $\mathbf{J}$ and Peterson DL (2007). Setting and Revising Antibacterial Susceptibility Breakpoints. Clinical Microbiology Reviews 20 (3): 391-408.

[22] Walsh C (2000) Molecular Mechanisms that Confer Antibacterial Drug Resistance. Nature 406: 775-781.

[23] Watts JL and Lindeman CJ (2006) Antimicrobial Susceptibility Testing of Bacteria of Veterinary Origin. In Aarestrup F, editors. Antimicrobial Resistance in Bacteria of Animal Origin. ASM Press, Washington, DC. 29-36.
[24] White DG, McDermott PF and Walker RD (2003) Antimicrobial Susceptibility Testing Methodologies. In Torrence ME and Isaacson RE, editors. Microbial food Safety in Animal Agriculture. Wiley online library. DOI: 10.1002/9780470752616.ch5.

[25] Baquero F and Blàazquez J (1997) Evolution of Antibiotic Resistance. Trends in Ecology and Evolution 12(12): 482-487.

[26] Witte W (2000) Selective Pressure by Antibiotic Use in Livestock. International Journal of Antimicrobial Agents 16: 519-524.

[27] Berger-Bächi B. 2002. Resistance Mechanisms of Gram Positive Bacteria. International Journal of Medical Microbiology. 292: 27-35.

[28] Papp-Wallace, K. M. et al., 2010. Inhibitor resistance in the KPC-2 beta-lactamase, a preeminent property of this class A beta-lactamase. Antimicrobial agents and chemotherapy, 54(2), pp. 890-7. Available at: http://www.pubmedcentral.nih.gov/articlerender.fcgi?artid=28 $12178 \&$ tool $=$ pmcentre $\&$ rendertype $=$ abstract.

[29] MacFaddin JF (2000) Biochemical Tests for Identification of Medical Bacteria, 3rd edition. Lippincott Williams \& Wilkins 912p.

[30] Centers for Disease Control and Prevention - Klebsiella Quotation: "Increasingly, Klebsiella bacteria have developed antimicrobial resistance, most recently to the class of antibiotics known as carbapenems.

[31] Dyar, O. J. et al., 2012. High prevalence of antibiotic resistance in commensal Escherichia coli among children in rural Vietnam. BMC infectious diseases, 12, p. 92.

[32] Depardieu F, Podglajen, Leclercq R, Collatz E and Courvalin P (2007) Modes and Modulations of Antibiotic resistance Gene Expression. Clinical Microbiology. 20 (1): 79-114.

[33] Fàbrega A, Sànchez-Cespedes J, Soto S and Vila Jordi (2008) Quinolone Resistance in the Food Chain. International Journal of Antimicrobial Agents. 31: 307-311.

[34] Cheng, W. et al., 2013. Abundance and persistence of antibiotic resistance genes in livestock farms: A comprehensive investigation in eastern China. Environment International, 61, pp. 1-7.

[35] Prescott JF (2006) Beta-lactam antibiotics: Penam penicillins. In Giguère S, Prescott JF, Baggot JD, Walker RD, Dowling $\mathrm{M}$, editors. Antimicrobial Therapy in Veterinary Medicine. Ames, IA: Blackwell Publishing. Page 124.

[36] Rinsky, J. L. et al., 2013. Livestock-Associated Methicillin and Multidrug Resistant Staphylococcus aureus Is Present among Industrial, Not Antibiotic-Free Livestock Operation Workers in North Carolina. PLoS ONE, 8 (7), pp. 1-11.

[37] Cordoba, G. et al., 2015. Prescribing style and variation in antibiotic prescriptions for sore throat: cross-sectional study across six countries. BMC family practice, 16, p. 7.

[38] Barnett, M. L. \& Linder, J. a, 2014. Antibiotic prescribing for adults with acute bronchitis in the United States, 1996-2010. JAMA, 311 (19), pp. 2020-2. 